\title{
Quantumness in decoherent quantum walk using measurement-induced disturbance
}

\author{
R. Srikanth, $, 1,2$, Subhashish Banerjee, 3 , 团 and C. M. Chandrashekar ${ }^{4,5}$, 团 \\ ${ }^{1}$ Poornaprajna Institute of Scientific Research, Devanahalli, Bangalore 562 110, India \\ ${ }^{2}$ Raman Research Institute, Sadashiva Nagar, Bangalore, India \\ ${ }^{3}$ Chennai Mathematical Institute, Siruseri, Chennai, India \\ ${ }^{4}$ Institute for Quantum Computing, University of Waterloo, Waterloo, Ontario, Canada N2L 3G1 \\ ${ }^{5}$ Center for Quantum Sciences, The Institute of Mathematical Sciences, Chennai 600113, India
}

\begin{abstract}
The classicalization of a decoherent discrete-time quantum walk on a line or an $n$-cycle can be demonstrated in various ways that do not necessarily provide a geometry-independent description. For example, the position probability distribution becomes increasingly Gaussian, with a concomitant fall in the standard deviation, in the former case, but not in the latter. As another example, each step of the quantum walk on a line may be subjected to an arbitrary phase gate, without affecting the position probability distribution, no matter whether the walk is noiseless or noisy. This symmetry, which is absent in the case of noiseless cyclic walk, but is restored in the presence of sufficient noise, serves as an indicator of classicalization, but only in the cyclic case. Here we show that the degree of quantum correlations between the coin and position degrees of freedom, quantified by a measure based on the disturbance induced by local measurements [Luo, Phys. Rev. A 77, 022301 (2008)], provides a suitable measure of classicalization across both type of walks. Applying this measure to compare the two walks, we find that cyclic quantum walks tend to classicalize faster than quantum walks on a line because of more efficient phase randomization due to the self-interference of the two counter-rotating waves. We model noise as acting on the coin, and given by the squeezed generalized amplitude damping (SGAD) channel, which generalizes the generalized amplitude damping channel.
\end{abstract}

\section{INTRODUCTION}

Quantum walks (QWs) are the quantum analog of the classical random walks (CRWs) [1 [5]. It is now well known that the quantum features of interference and superposition make QW spread quadratically faster than CRW. Like their classical counterpart, QWs are also widely studied in two forms: continuous-time QW (CTQW) [5] and discretetime QW (DTQW) [3, 4, 6, 7] and are found to be very useful from the perspective of quantum algorithms [8 11]. Furthermore, they have been used to demonstrate the coherent quantum control over atoms, quantum phase transition [12], to explain phenomena such as the breakdown of an electric-field driven system [13] and direct experimental evidence for wavelike energy transfer within photosynthetic systems [14, 15]. Experimental implementation of QWs has been reported with samples in an nuclear magnetic resonance (NMR) system 16, 17], in the continuous tunneling of light fields through waveguide lattices [18], in the phase space of trapped ions [19, 20] based on the scheme proposed by [21], with single neutral atoms in optically trapped atoms 22], and with single photons [23]. Recently, implementation of a QW-based search algorithm in an NMR system has been reported [24]. Various other schemes have been proposed for their physical realization in different physical systems 25,28$]$.

Decoherence in QW and the transition of QW to CRW is quite important from the viewpoint of practical implementation, and has been studied by various authors 29 34]. In particular, in Refs. 32, 33], we investigated some qualitatively different ways in which environmental effects suppress quantum superposition in QW on a line and an $n$ - cycle. In these works, we have modeled noise as the bit flip channel, the phase flip channel (decoherence without dissipation), and the generalized amplitude damping channel (decoherence with dissipation), which are of relevance to studies in quantum optics and condensed matter systems. The latter type of noise in the QW context appears to have been first studied by us [32]. Here we employ a more general noise acting on the coin, the squeezed generalized amplitude damping (SGAD) channel, that arises due to coupling with a squeezed, thermal bath [35].

In this work, we consider the issue of quantifying the degree of quantumness of the walker during the transition from a pure QW dynamics to the CRW dynamics. As a general problem applied to an arbitrary quantum system, this is still not fully understood. Intuitively, one would expect that quantum entanglement captures the entire content of quantum correlations in a system, but this may not be so, in view of other contributions, coming from superposition

\footnotetext{
*Electronic address: srik@poornaprajna.org

${ }^{\dagger}$ Electronic address: subhashish@cmi.ac.in

‡Electronic address: cmadaiah@iqc.ca ; chandru@imsc.res.in
} 
and noncommutativity of observables. In Ref. [36], a quantity called 'discord', which is defined as the difference between two different quantum generalizations of classical mutual information, is shown to be a viable measure of the quantumness of correlations in a bipartite system. One can produce systems in which discord is nonvanishing even though entanglement vanishes. However, computing discord in a large-dimensional system can be difficult because it requires an optimization over all possible local measurement strategies. An alternative method is via 'dissonance', defined as the relative entropy between a separable state $\sigma$ and the closest classical state (a separable state that is a mixture of locally distinguishable states), where $\sigma$ is such that it has the smallest relative entropy of entanglement with the given (in general, entangled) state $\rho$ [37]. Therefore, unlike discord, dissonance is a measure of quantumness that excludes entanglement. An operational approach that employs measurement-induced disturbance as a measure of quantumness has been proposed by Luo [38]. In this work, we use this 'quantumness via measurement-induced disturbance' (QMID) between the coin and position degrees of freedom to study the effect of decoherence on QW.

This article is arranged as follows. In Sec. II we describe the DTQW model on a line and an $n-c y c l e$. In Sec. III we introduce the SGAD channel as the process that decoheres the walk, and discuss some signatures of the classicalization of QW. In Sec. IV] we discuss a specific kind of walk symmetry as a means to characterize the loss of quantumness in a decoherent walk, and point out its drawbacks. In Sec. V we investigate QMID as a means to quantify quantumness of the correlations between the coin and the position degrees of freedom, that is applicable to decoherent QWs both on a line and on an $n$-cycle. Finally, we conclude in Sec. VI.

\section{DISCRETE-TIME QUANTUM WALK ON A LINE AND AN $n$-CYCLE}

The DTQW in one dimension is modeled as a particle consisting of a two-level coin (a qubit) living in the Hilbert space $\mathcal{H}_{c}$, spanned by $|0\rangle$ and $|1\rangle$, and a position degree of freedom living in the Hilbert space $\mathcal{H}_{p}$, spanned by $\left|\psi_{x}\right\rangle$, where $x \in \mathbb{I}$, the set of integers. In an $n$-cycle walk, there are only $n$ allowed positions, and in addition the periodic boundary condition $\left|\psi_{x}\right\rangle=\left|\psi_{x \bmod n}\right\rangle$ is imposed. A $t$-step coined QW is generated by iteratively applying a unitary operation $W$ which acts on the Hilbert space $\mathcal{H}_{c} \otimes \mathcal{H}_{p}$ :

$$
\left|\Psi_{t}\right\rangle=W^{t}\left|\Psi_{i n}\right\rangle
$$

where $\left|\Psi_{i n}\right\rangle=\left(\cos (\delta / 2)|0\rangle+\sin (\delta / 2) e^{i \phi}|1\rangle\right) \otimes\left|\psi_{0}\right\rangle$ is an arbitrary initial state of the particle and $W \equiv U(B \otimes \mathbb{1})$, where $U(2) \ni B=B_{\xi, \theta, \zeta} \equiv\left(\begin{array}{cc}e^{i \xi} \cos (\theta) & e^{i \zeta} \sin (\theta) \\ e^{-i \zeta} \sin (\theta) & -e^{-i \xi} \cos (\theta)\end{array}\right)$ (with components denoted $B_{j k}$ ) is the coin operation. $U$ is the controlled-shift operation

$$
U \equiv|0\rangle\left\langle 0\left|\otimes \sum_{x}\right| \psi_{x}-1\right\rangle\left\langle\psi_{x}|+| 1\right\rangle\left\langle 1\left|\otimes \sum_{x}\right| \psi_{x}+1\right\rangle\left\langle\psi_{x}\right| .
$$

For an $n$-cycle $U$ is replaced by

$$
U^{c} \equiv|0\rangle\left\langle 0\left|\otimes \sum_{x=0}^{n-1}\right| \psi_{x-1} \bmod n\right\rangle\left\langle\psi_{x}|+| 1\right\rangle\left\langle 1\left|\otimes \sum_{x=0}^{n-1}\right| \psi_{x+1} \bmod n\right\rangle\left\langle\psi_{x}\right|
$$

The probability to find the particle at site $x$ after $t$ steps is given by $p(x, t)=\left\langle\psi_{x}\left|\operatorname{tr}_{c}\left(\left|\Psi_{t}\right\rangle\left\langle\Psi_{t}\right|\right)\right| \psi_{x}\right\rangle$.

\section{DECOHERENCE VIA SQUEEZED GENERALIZED AMPLITUDE DAMPING CHANNEL}

A QW implemented on a quantum computer is inevitably affected by errors caused by noise due to the environment. Some physically relevant models of noise are as follows: a phase flip channel (which is equivalent to a phase damping or purely dephasing channel), a bit flip channel, a generalized amplitude damping channel $(T \geq 0)$ and an SGAD channel, which is used here. Our numerical implementation of these channels evolves the density matrix at each walk step employing the Kraus operator representation for them.

The physical origin and the effect of various types of noise, such as phase flip, bit flip, amplitude and generalized amplitude damping, on the DTQW have been studied in detail in Refs. [32, 33]. Here we study the behavior of decoherent QW subjected to a recently introduced noise process, the SGAD channel [35], which we briefly discuss below for completeness. 
System evolution in the interaction picture, for the case of SGAD, has the following form [39, 40]:

$$
\begin{aligned}
\frac{d}{d t} \rho^{s}(t) & =\gamma_{0}(N+1)\left(\sigma_{-} \rho^{s}(t) \sigma_{+}-\frac{1}{2} \sigma_{+} \sigma_{-} \rho^{s}(t)-\frac{1}{2} \rho^{s}(t) \sigma_{+} \sigma_{-}\right) \\
& +\gamma_{0} N\left(\sigma_{+} \rho^{s}(t) \sigma_{-}-\frac{1}{2} \sigma_{-} \sigma_{+} \rho^{s}(t)-\frac{1}{2} \rho^{s}(t) \sigma_{-} \sigma_{+}\right) \\
& -\gamma_{0} M \sigma_{+} \rho^{s}(t) \sigma_{+}-\gamma_{0} M^{*} \sigma_{-} \rho^{s}(t) \sigma_{-} .
\end{aligned}
$$

Here $\gamma_{0}=\left(4 \omega^{3}|\vec{d}|^{2}\right) /\left(3 \hbar c^{3}\right)$ is the spontaneous emission rate, while $\sigma_{+}, \sigma_{-}$are the standard raising and lowering operators. Also, $N=N_{\mathrm{th}}\left[\cosh ^{2}(r)+\sinh ^{2}(r)\right]+\sinh ^{2}(r), M=-\frac{1}{2} \sinh (2 r) e^{i \Phi}\left(2 N_{\mathrm{th}}+1\right)$, where $N_{\mathrm{th}}=1 /\left(e^{\hbar \omega_{0} / k_{B} T}-1\right)$ is the Planck distribution giving the number of thermal photons at frequency $\omega_{0}$, while $r, \Phi$ are the bath squeezing parameters [41].

The SGAD channel is generated by the master Eq. (4), with nonvanishing $r, \Phi, T$. The Kraus operators for this channel, depicting the system-bath interaction for a time $t=\Delta$ are [35]

$$
\begin{array}{ll}
E_{0} \equiv \sqrt{p_{1}}\left[\begin{array}{ll}
\sqrt{1-\alpha(\Delta)} & 0 \\
0 & 1
\end{array}\right] ; & E_{1} \equiv \sqrt{p_{1}}\left[\begin{array}{ll}
0 & 0 \\
\sqrt{\alpha(\Delta)} & 0
\end{array}\right] ; \\
E_{2} \equiv \sqrt{p_{2}}\left[\begin{array}{lll}
\sqrt{1-\mu(\Delta)} & 0 \\
0 & \sqrt{1-\nu(\Delta)}
\end{array}\right] ; & E_{3} \equiv \sqrt{p_{2}}\left[\begin{array}{ll}
0 & \sqrt{\nu(\Delta)} \\
\sqrt{\mu(\Delta)} e^{-i \theta(\Delta)} & 0
\end{array}\right] .
\end{array}
$$

Here,

$$
\begin{gathered}
\nu(\Delta)=\frac{N}{p_{2}(2 N+1)}\left(1-e^{-\gamma_{0}(2 N+1) \Delta}\right), \\
\mu(\Delta)=\frac{2 N+1}{2 p_{2} N} \frac{\sinh ^{2}\left(\gamma_{0} a \Delta / 2\right)}{\sinh \left(\gamma_{0}(2 N+1) \Delta / 2\right)} \exp \left(-\frac{\gamma_{0}}{2}(2 N+1) \Delta\right), \\
\alpha(\Delta)=\frac{1}{p_{1}}\left(1-p_{2}[\mu(\Delta)+\nu(\Delta)]-e^{-\gamma_{0}(2 N+1) \Delta}\right), \\
p_{1}+p_{2}=1, \\
p_{2}(\Delta)=\frac{1}{(A+B-C-1)^{2}-4 D} \times\left\{A^{2} B+C^{2}+A\left[B^{2}-C-B(1+C)-D\right]-(1+B) D-C(B+D-1)\right. \\
\pm 2 \sqrt{D[B-A B+(A-1) C+D][A-A B+(B-1) C+D]}\}
\end{gathered}
$$

where $A=\frac{2 N+1}{2 N} \frac{\sinh ^{2}\left(\gamma_{0} a \Delta / 2\right)}{\sinh \left(\gamma_{0}(2 N+1) \Delta / 2\right)} \exp \left(-\gamma_{0}(2 N+1) \Delta / 2\right), B=\frac{N}{2 N+1}\left(1-\exp \left(-\gamma_{0}(2 N+1) \Delta\right)\right), C=A+B+$ $\exp \left(-\gamma_{0}(2 N+1) \Delta\right), D=\cosh ^{2}\left(\gamma_{0} a \Delta / 2\right) \exp \left(-\gamma_{0}(2 N+1) \Delta\right)$.

The SGAD channel extends the concept of a generalized amplitude damping (GAD) channel, which arises due to the dissipative interaction with a purely thermal bath to that with a squeezed thermal bath. One recovers generalized amplitude damping channel, if the squeezing parameter $r \rightarrow 0$.

Figure 1(a) depicts the gradual classicalization of a $\mathrm{QW}$ on a line under the action of the SGAD noise for different channel parameters. It is seen that, as the QW turns into a CRW, correspondingly, the probability distribution becomes increasingly Gaussian, causing the quadratic functional dependence of variance on time, characteristic of quantum behavior, to become linear ( cf. Fig. 2 of Ref. [30], and the analytical results presented therein). This pattern of quantum-to-classical transition has also been observed experimentally, in discrete-time quantum walks using single-photon, subjected to decoherence of the pure dephasing type [23]. This behavior is quite generic, and can be shown, for example, to arise under arbitrary Markovian decoherence of a continuous-time quantum walk on a graph (cf. Fig. 3 of Ref. [42]). The Gaussianization is directly reflected in the fall of standard deviation, depicted in Fig. 1(b) because classicalization causes probability to cluster closer to the mean position. As it turns out, neither the Gaussianization, nor the concomitant dramatic drop in standard deviation, happens in the case of QW on an $n$-cycle [Fig. 2(a)]. Thus the characterization of loss of quantumness in a QW in terms of shape change of the probability distribution according to this pattern is, while convenient for walk on a line, not fruitful in the case of cyclic walk. In the following section, we will discuss why a sort of 'gauge freedom' in QWs also is not an appropriate geometry-independent measure of classicalization of QW. 


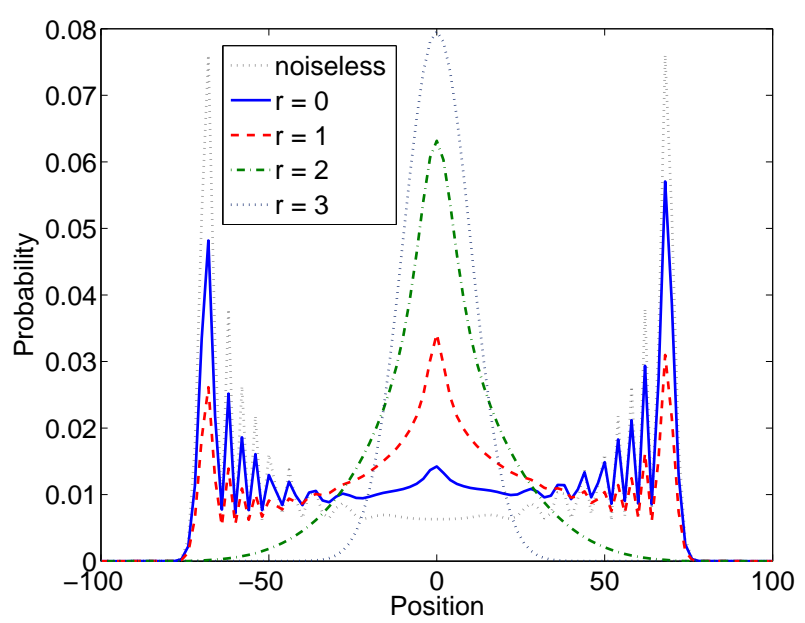

(a)

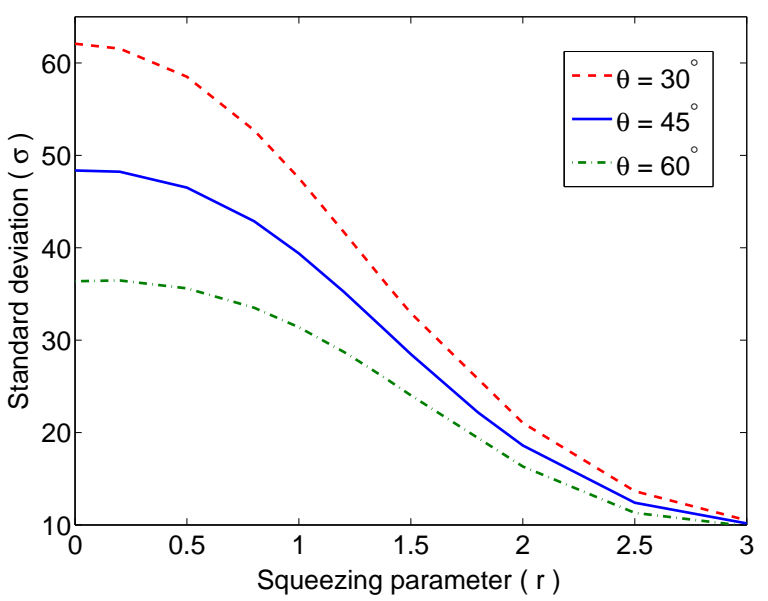

(b)

FIG. 1: The effect of increasing the environmental squeezing parameter of the SGAD channel, on the position probability distribution of a particle with the initial state $(1 / \sqrt{2})(|0\rangle+i|1\rangle) \otimes\left|\psi_{0}\right\rangle$ and coin toss instruction given by $B\left(0^{\circ}, 45^{\circ}, 0^{\circ}\right)$. Other channel parameters are fixed at $T=2, \gamma_{0}=0.025$ and $\Delta=0.1$. (a) The probability distribution on a line for different values of $r$, at time $t=100$ steps. With increasing noise (parametrized by $r$ ), the distribution transforms from the characteristic QW twin-peaked distribution to the classical Gaussian. (b) The Gaussianization is evidently accompanied by a reduction in the standard deviation, as seen here. The plots are given for different values of the coin toss parameter $\theta$.

\section{A SYMMETRY OF QUANTUM WALK ON A LINE}

We will call an operation applied at each step of the walk that leaves the position probability distribution $p(x, t)$ of the particle unchanged as 'walk symmetry'. The phase gate $G(\alpha)=\left(\begin{array}{ll}1 & 0 \\ 0 & e^{i \alpha}\end{array}\right)$ acting on the coin is such a walk symmetry for walk on line, but not for that on an $n$-cycle. The physical significance of $G$ is that it helps identify a family of QWs that are equivalent from the viewpoint of physical implementation, which can sometimes allow a significant practical simplification [32].

Suppose the walker begins in the state $(a|0\rangle+b|1\rangle) \otimes\left|\psi_{0}\right\rangle$, with $|a|^{2}+|b|^{2}=1$. If the coin operator is denoted by the matrix $B \equiv\left\{B_{j, k}\right\}$, then after application of this operation followed by the conditional shift, the walker is in the state $\sum_{j_{1}}\left(a B_{j_{1} 0}+b B_{j_{1} 1}\right)\left|j_{1}, \psi_{2 j_{1}-1}\right\rangle$, where $j_{1} \in\{0,1\}$. Proceeding thus, after $t$ steps the walker on a line and on an $n$-cycle are seen to be, respectively,

$$
\begin{aligned}
\left|\Psi_{L}(t)\right\rangle & =\sum_{j_{1}, j_{2}, \cdots, j_{t}} e^{i J_{t} \alpha} B_{j_{t}, j_{t-1}} \cdots B_{j_{2}, j_{1}}\left(B_{j_{1} 0} a+B_{j_{1} 1} b\right)\left|j_{t}, \psi_{\left[2 J_{t}-t\right]}\right\rangle, \\
\left|\Psi_{C}(t)\right\rangle & \left.\left.=\sum_{j_{1}, j_{2}, \cdots, j_{t}} e^{i J_{t} \alpha} B_{j_{t}, j_{t-1}} \cdots B_{j_{2}, j_{1}}\left(B_{j_{1} 0} a+B_{j_{1} 1} b\right) \mid j_{t}, \psi_{\left[2 J_{t}-t\right.} \bmod n\right]\right\rangle,
\end{aligned}
$$

where $J_{t}=j_{1}+j_{2}+\cdots+j_{t}$ and $0 \leq J_{t} \leq t$. The projection of the state $\left|\Psi_{L}(t)\right\rangle$, Eq. (5a onto some position state $\left|\psi_{x}\right\rangle \equiv\left|\psi_{2 J_{t}-t}\right\rangle$ is given by

$$
\begin{aligned}
\left\langle\psi_{x} \mid \Psi_{L}(t)\right\rangle & =\sum_{j_{1}, j_{2}, \cdots, j_{t}} e^{i \alpha(x+t) / 2} B_{j_{t}, j_{t-1}} \cdots B_{j_{2}, j_{1}}\left(B_{j_{1} 0} a+B_{j_{1} 1} b\right)\left|j_{t}\right\rangle \\
& =\sum_{j_{1}, j_{2}, \cdots, j_{[t-1]}}\left(e^{i \alpha(x+t) / 2} B_{0, j_{t-1}} \cdots B_{j_{2}, j_{1}}\left(B_{j_{1} 0} a+B_{j_{1} 1} b\right)|0\rangle\right. \\
& +\left(e^{i \alpha[-1+(x+t) / 2]} B_{1, j_{t-1}} \cdots B_{j_{2}, j_{1}}\left(B_{j_{1} 0} a+B_{j_{1} 1} b\right)|1\rangle\right),
\end{aligned}
$$

where we have used the fact that $J_{t}=J_{t-1}+j_{t}=(x+t) / 2$. Therefore the probability to detect the particle at $x$ is 


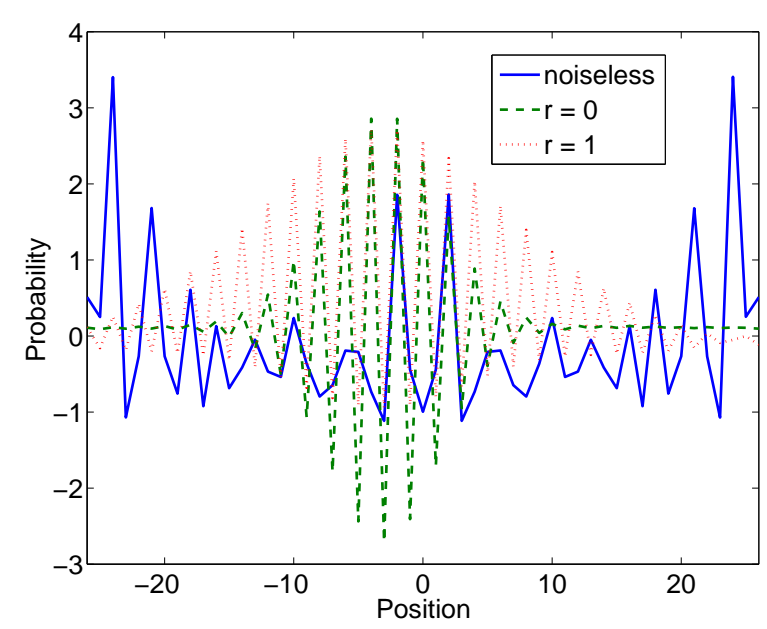

(a)

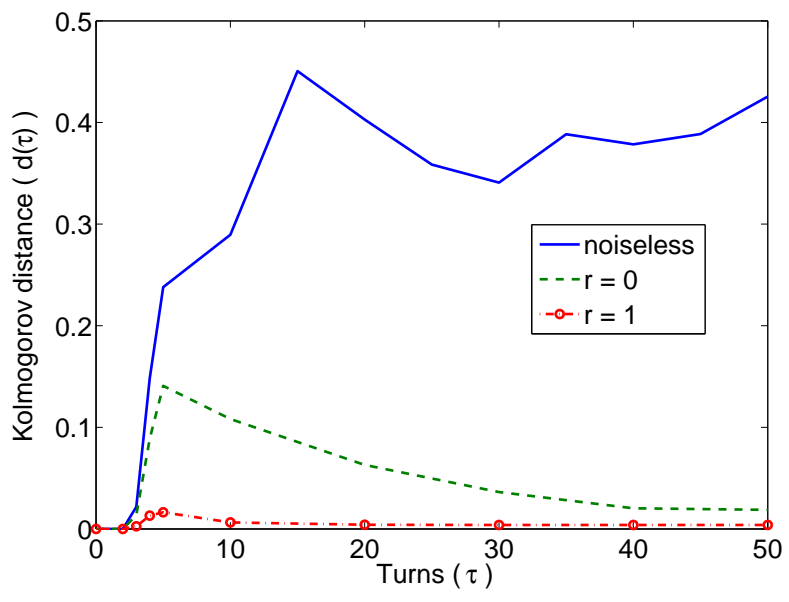

(b)

FIG. 2: (a) Probability distribution of a particle with the initial state $(1 / \sqrt{2})(|0\rangle+i|1\rangle) \otimes\left|\psi_{0}\right\rangle$ and coin toss instruction given by $B\left(0^{\circ}, 45^{\circ}, 0^{\circ}\right)$ on an $n$-cycle $(n=51)$ with increase in value of the $r$ for $\tau=100$. In contrast to the case of QW on a line [Figs. 1(a)] and 1(b)], note that the classicalized walk does not become Gaussian. All plots have been shifted by the respective mean value, and downscaled by a factor equal to the respective standard deviation, in order to bring out the shape change in the probability distributions. (b) The breakdown in 'gauge symmetry' is described by nonvanishing Kolmogorov (trace) distance between the probability distributions resulting from the nonapplication and application of a phase gate, $G\left(30^{\circ}\right)$ at each step of the cyclic walk [33]. The unitary walk is marked by a persistent, nonvanishing symmetry breakdown. Noise causes the gradual suppression of superposition terms, leading to a restoration of the symmetry.

given by:

$$
\left|\sum_{j_{1}, j_{2}, \cdots, j_{t-1}} B_{0, j_{t-1}} \cdots B_{j_{2}, j_{1}}\left(B_{j_{1} 0} a+B_{j_{1} 1} b\right)\right|^{2}+\left|\sum_{j_{1}, j_{2}, \cdots, j_{t-1}} B_{1, j_{t-1}} \cdots B_{j_{2}, j_{1}}\left(B_{j_{1} 0} a+B_{j_{1} 1} b\right)\right|^{2},
$$

independent of $\alpha$, whence the symmetry. Proceeding similarly in the case of QW on an $n$-cycle, we find that whereas each superposition term accumulates phase in the same way, the spatial spread of the state is restricted by the geometry not to exceed $n$, leading to Eq. (5b). As a result, fixing $x$ fixes $J_{t} \bmod n=(x+t) / 2 \bmod n$, but not $J_{t}$ itself, so that the phase terms in the superposition Eq. (5b) do not factor out globally in the above manner as with QW on a line, whence the breakdown in the symmetry in the cyclic case [Fig. 2(b)]. We use the notation $n=2 s+1$, where $s$ is the number of vertices on the left or right arm of the cycle, excluding the origin. We define turns $\tau$ by $t=\tau s$, where $t$ is walk time or the number of steps. Physically, the breakdown in symmetry can be attributed to interference of the clockwise and anticlockwise rotating waves on the cycle.

In Eq. (5a), note that the proof of symmetry works just the same even if the $B$ 's in each operation are distinct operators, and, further, even if they are arbitrary matrices, not necessarily unitary. With this insight, consider any CP map represented by the set of Kraus operators $\left\{E_{1}, \cdots, E_{d}\right\}$. The operators $E_{j}$ may be unitary, as with a Pauli channel like bit flip or phase flip channels, or not, as with the amplitude damping channel or squeezed generalized amplitude damping channel. Consider an $n$-fold application of the noise process represented by this CP map on an initial pure state $\rho \equiv\left|\Psi_{i n}\right\rangle\left\langle\Psi_{i n}\right|$. This produces

$$
\rho^{\prime}=\sum_{i_{1}, \cdots, i_{n}=1}^{d} E_{i_{n}} \cdots E_{i_{1}} \rho E_{i_{1}}^{\dagger} \cdots E_{i_{n}}^{\dagger}
$$

The state $\rho^{\prime}$ can equally well be regarded as a uniform mixture of the $d^{n}$ unnormalized states of the form $E_{i_{n}} \cdots E_{i_{1}}\left|\Psi_{i n}\right\rangle$, where a particular sequence $\mathbf{E}_{k} \equiv E_{i_{n}} \cdots E_{i_{1}}$ can be regarded as a 'noise trajectory'. Note that the $\left\{\mathbf{E}_{k}\right\}$ are themselves Kraus operators for they can be shown to be positive and to satisfy

$$
\sum_{k=1}^{d^{n}} \mathbf{E}_{k}^{\dagger} \mathbf{E}_{k}=\mathbb{I}
$$




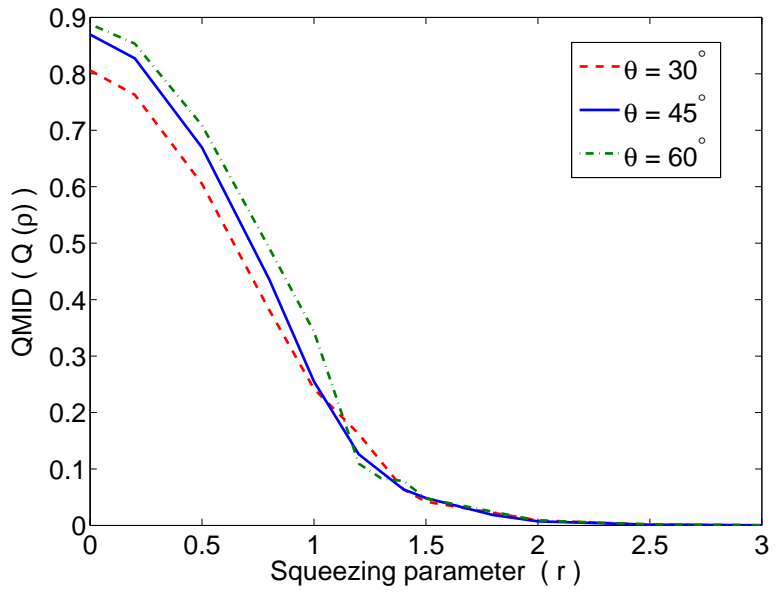

(a)

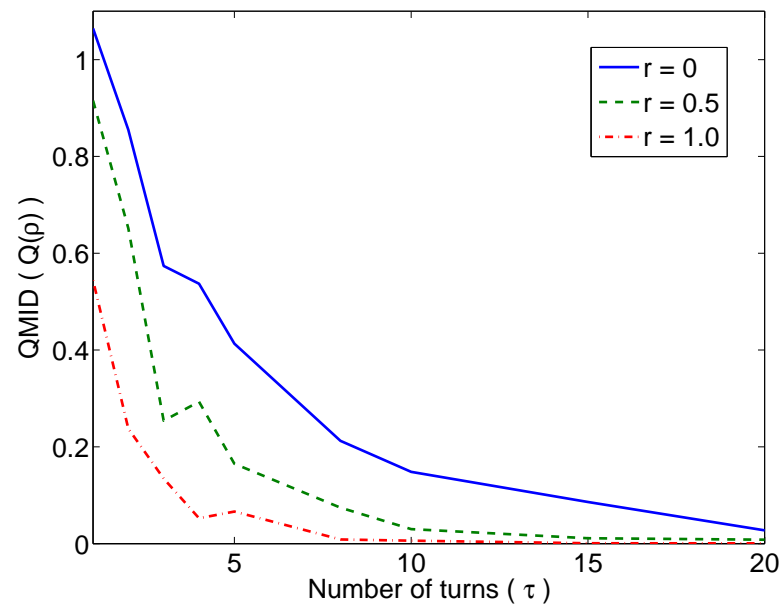

(b)

FIG. 3: (a) Decrease in QMID with increasing squeezing parameter $r$ for a particle walking on a line at time $t=100$ steps, with the initial state $(1 / \sqrt{2})(|0\rangle+i|1\rangle) \otimes\left|\psi_{0}\right\rangle$ and coin toss instruction given by $B\left(0^{\circ}, \theta, 0^{\circ}\right)$, where $\theta=30^{\circ}, \theta=45^{\circ}$, and $\theta=60^{\circ}$. A similar decreasing pattern can also be seen in the cyclic case. Note the slight $\theta$ dependence of $Q$, and the switch in this dependence around $r=1.2$. (b) Variation of QMID $[Q(\rho)]$ for QW on an $n-$ cycle $(n=101)$, with increase in number of turns $(\tau)$ of the cycle. With increase in $r, Q(\rho)$ decreases faster, implying more rapid classicalization. Other environmental parameters are $\gamma_{0}=0.025$, temperature $(T=2)$, and $\Delta=0.1$.

A particular noise trajectory of $n$ steps of a walk, given by $(U B)^{n}\left|\Psi_{i n}\right\rangle$ could be, for example, $\left(U B E_{i_{n}}\right)\left(U B E_{i_{n-1}}\right) \cdots\left(U B E_{i_{2}}\right)\left(U B E_{i_{1}}\right)\left|\Psi_{i n}\right\rangle$, where the noise is assumed to act on the coin just before the application of $B$ at each step. This can be rewritten, matrix-multiplying $B$ with the noise operator, as $\mathbf{E}_{x}\left|\Psi_{i n}\right\rangle \equiv$ $\left(U B_{i_{n}}^{\prime}\right)\left(U B_{i_{n-1}}^{\prime}\right) \cdots\left(U B_{i_{2}}^{\prime}\right)\left(U B_{i_{1}}^{\prime}\right)\left|\Psi_{i n}\right\rangle$. If the $B$ 's in Eq. (5a) are replaced by the $B^{\prime}$ 's, we are led by the same reasoning to conclude that along each trajectory, $p(x, t)$ is unaffected by the application of $G(\alpha)$. Hence, the average overall $d^{n}$ noise trajectories will also leave $p(x, t)$ unaffected.

We assumed above that the noise acts at each step just before the coin operation, but it is clear that it may equally well act just after the coin operation. Furthermore, it may also act after the shift operation. To see this we rewrite, using the associative property of quantum operations, a particular noise trajectory $\left(E_{i_{n}} U B\right)\left(E_{i_{n-1}} U B\right) \cdots\left(E_{i_{2}} U B\right)\left(E_{i_{1}} U B\right)\left|\Psi_{L}\right\rangle$ as $E_{i_{n}}\left(U B E_{i_{n-1}}\right)\left(U B \cdots E_{i_{2}}\right)\left(U B E_{i_{1}}\right) U B\left|\Psi_{i n}\right\rangle$, then as $E_{i_{n}}\left(U B_{i_{n-1}}^{\prime}\right)\left(\cdots U B_{i_{2}}^{\prime}\right)\left(U B_{i_{1}}^{\prime}\right) U B\left|\Psi_{L}\right\rangle$ and finally as $E_{i_{n}}\left(U B E_{i_{n-1}}\right)\left(U B \cdots E_{i_{2}}\right)\left(U B E_{i_{1}}\right)\left|\Psi_{L}^{\prime}\right\rangle$ and once again the above reasoning can be applied to show that symmetry holds even with this model of noise. In earlier works, we explicitly verified this for the case when the noise is a phase damping channel [32] and a generalized amplitude damping channel [33]. Not surprisingly, noise restores symmetry for the cyclic QW, which can be understood simply as the result of suppression of quantum superpositions [33].

Figure 2(b) depicts the breakdown of the symmetry in noiseless QW on an $n$-cycle, and its restoration with the application of noise. At each time step, symmetry breakdown is quantified as the trace or Kolmogorov distance [43] between the two probability distributions obtained by applying or not applying the phase gate on every step. We note that while $\tau \leq 2$, the cyclic walk remains symmetric, as the diffusing particle is 'unaware' of its nontrivial topology. From Eq. (5b), we see that the beginning of breakdown is when $\max \left(2 J_{t}-t\right) \geq n$, which happens when $t \geq n$, or $\tau \geq 2+(1 / s)$. Clearly, even though the symmetry breakdown is potentially a good probe of walk topology, as an indicator of quantumness, it is relevant only to cyclic walk, and thus not a geometry-independent indicator of classicalization.

\section{QUANTUMNESS USING MEASUREMENT-INDUCED DISTURBANCE}

Finally we consider the classicalization of QW on a line and an $n$-cycle under the influence of the SGAD channel, using QMID as a measure of quantumness of the correlations between the coin and position degrees of freedom. Given a bipartite state $\rho$ living in the Hilbert space $\mathcal{H}_{A} \otimes \mathcal{H}_{B}$, and the reduced density matrices being denoted by $\rho_{A}$ and 


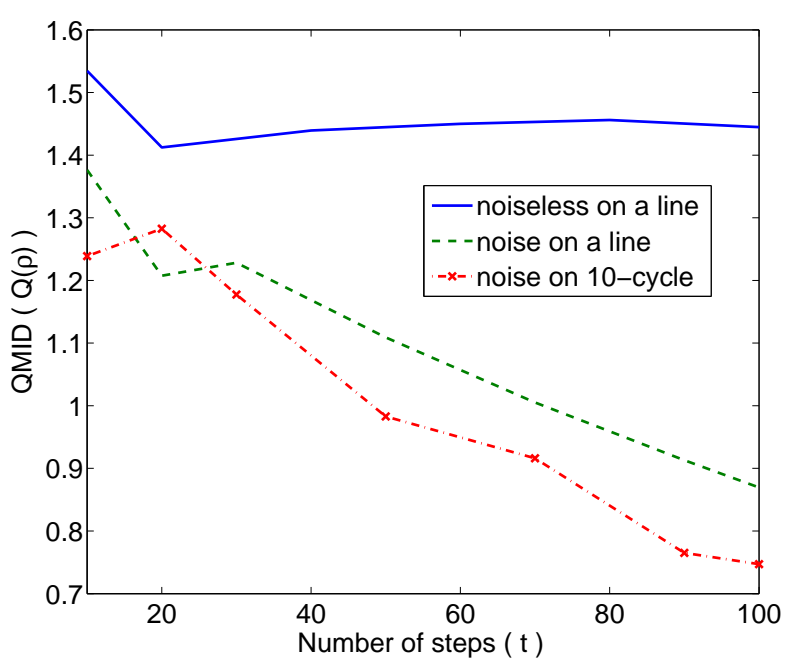

(a)

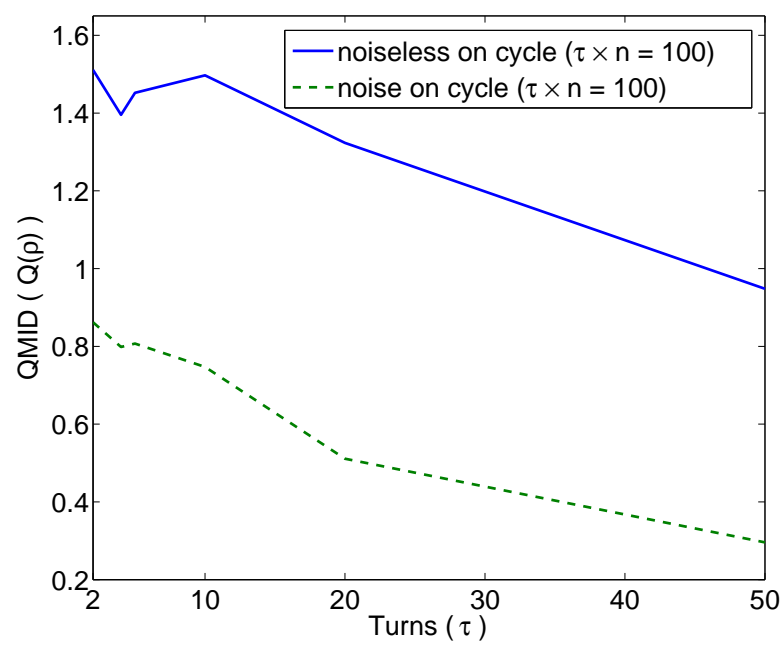

(b)

FIG. 4: A comparison of the degree of classicalization between QW on a line and that on an $n$-cycle, via QMID as the measure of quantumness. (a) For a fixed setting of the environment-induced decoherence (here $T=0.1, \gamma_{0}=0.05, \Delta=0.1$ and $r=0)$ and any given sufficiently large time, the cyclic walk typically becomes classical more rapidly on account of enhanced randomization due to the interference of the counter-rotating waves. (b) This is evidenced by the suppression of QMID with increase in the number of turns $\tau$, keeping time $t$ and all noise parameters fixed.

$\rho_{B}$, a reasonable measure of total correlations between systems $A$ and $B$ is the mutual information:

$$
I(\rho)=S\left(\rho_{A}\right)+S\left(\rho_{B}\right)-S(\rho)
$$

where $S(\cdot)$ denotes von Neumann entropy. If $\rho_{A}=\sum_{j} p_{A}^{i} \Pi_{A}^{i}$ and $\rho_{B}=\sum_{j} p_{B}^{i} \Pi_{B}^{i}$, then the measurement induced by the spectral resolution of the reduced states is

$$
\Pi(\rho) \equiv \sum_{j, k} \Pi_{A}^{j} \otimes \Pi_{B}^{k} \rho \Pi_{A}^{j} \otimes \Pi_{B}^{k}
$$

The state $\Pi(\rho)$ may be considered classical in the sense that there is a (unique) local measurement strategy, namely $\Pi$, that leaves $\Pi(\rho)$ unchanged. This strategy is special in that it produces a classical state in $\rho$ while keeping the reduced states invariant.

According to Luo [38], we may consider as a measure of quantum correlations the quantity $D[\rho, \Pi(\rho)]$, where $D$ is a suitable measure of quantum distance, such as Hilbert-Schmidt distance or Bures measure. If we accept that $I[\Pi(\rho)]$ is a good measure of classical correlations in $\rho$, then one may consider QMID, given by

$$
Q(\rho)=I(\rho)-I[\Pi(\rho)],
$$

as a reasonable measure of quantum correlation 38].

Our application of QMID to QW to derive the quantumness of coin-position correlations supports this view. We numerically determined $\rho$ in Eq. (77), by applying SGAD noise at each walk step, for various channel parameters like temperature $T$, coupling constant $\gamma_{0}, \Delta$, and environmental squeezing parameter $r$. The operator $\Pi$ in Eq. (8), and thence $Q(\rho)$ in Eq. (9), are obtained by diagonalization of the reduced density matrices $\rho_{A}$ and $\rho_{B}$.

Figure 3 presents typical plots of QMID in a QW system subjected to a SGAD channel with different variable parameters. In Fig. 3(a), we depict the fall of QMID in noisy QW on a line with increasing environment squeezing $r$. A similar pattern emerges also in the case of cyclic QW. Figure 3(b) depicts the fall of QMID in a noisy, cyclic QW with increasing time, a pattern that is seen also in the case of $\mathrm{QW}$ on a line. Both figures demonstrate, as expected, that increasing the level of noise (parametrized by $r$ ) is detrimental to the degree of quantumness. More generally, these findings suggest that QMID provides the desired geometry-independent way to describe the classicalization of QW, unlike the previously discussed ways.

With this result, we may compare the decohering properties of QW on a line with those of cyclic QW (Fig. 4). For fixed noise setting, at any given sufficiently large time, as seen from Fig. 4(a) cyclic walk is more classical 
than walk on the line, which may be understood as follows. Decoherence randomizes phase, and in cyclic walk, the self-interference of the two oppositely rotating waves enhances this randomization. This explanation is supported by Fig. 4(b) where increasing the number of turns $\tau$ keeping time $t$ and all noise parameters fixed is seen to suppress the quantumness.

\section{CONCLUSIONS}

In this work, we investigated the loss of quantumness in a decoherent DTQW on a line and an $n$-cycle. We showed that QMID provides a reasonable geometry-independent description of the degree of classicalization of the walk, unlike such familiar criterion as the Gaussianization of the walk probability distribution, which applies only to walk on a line, or a specific symmetry principle, which applies only to cyclic walks. The noise is modeled as an SGAD channel acting on the coin. One might consider the amount of entanglement between the coin and the position degrees of freedom as a measure of quantumness, but, as mentioned in the Sec. I. this might be an underestimate because it ignores the nonclassicality due to non-commutative observable algebra of the coin and position degrees of freedom. Quantum discord is a transparent measure of quantumness, but its computation for large systems like a quantum walker can be hard because of the optimization it involves. This suggests that QMID is a suitable, geometry-independent operational tool to describe the quantumness of QW. As a first application of this idea, we showed that cyclic QWs tend to classicalize faster than QWs on a line on account of more efficient phase randomization.

[1] G. V. Riazanov, Sov. Phys. JETP 61107 (1958).

[2] R. P. Feynman and A.R. Hibbs, Quantum Mechanics and Path Integrals (McGraw-Hill, New York, 1965).

[3] Y. Aharonov, L. Davidovich and N. Zagury, Phys. Rev. A 48, 1687, (1993).

[4] D. A. Meyer, J. Stat. Phys. 85, 551 (1996).

[5] E. Farhi and S. Gutmann, Phys.Rev. A 58, 915 (1998).

[6] A. Ambainis, E. Bach, A. Nayak, A. Vishwanath and J. Watrous, Proceeding of the 33rd ACM Symposium on Theory of Computing (ACM Press, New York, 2001), p.60.

[7] A. Nayak and A. Vishwanath, DIMACS Technical Report, No. 2000-43 (2001) ; arXiv:quant-ph/0010117.

[8] A. Ambainis, Int. Journal of Quantum Information, 1, No.4, 507-518 (2003).

[9] A. M. Childs, R. Cleve, E. Deotto, E. Farhi, S. Gutmann and D. A. Spielman, in Proceedings of the 35th ACM Symposium on Theory of Computing (ACM Press, New York, 2003), p.59.

[10] N. Shenvi, J. Kempe and K. Birgitta Whaley, Phys. Rev. A 67, 052307, (2003).

[11] A. Ambainis, J. Kempe, and A. Rivosh, Proceedings of ACM-SIAM Symp. on Discrete Algorithms (SODA), (AMC Press, New York, 2005), pp.1099-1108.

[12] C. M. Chandrashekar and R. Laflamme, Phys. Rev. A 78, 022314 (2008).

[13] T. Oka, N. Konno, R. Arita, and H. Aoki, Phys. Rev. Lett. 94, 100602 (2005).

[14] G. S. Engel et. al., Nature 446, 782-786 (2007).

[15] M. Mohseni, P. Rebentrost, S. Lloyd, A. Aspuru-Guzik, J. Chem. Phys. 129, 174106 (2008).

[16] J. Du, H. Li, X. Xu, M. Shi, J. Wu, X. Zhou, and R. Han, Phys. Rev. A 67, 042316 (2003)

[17] C. A. Ryan, M. Laforest, J. C. Boileau, and R. Laflamme, Phys. Rev. A 72, 062317 (2005).

[18] H. B. Perets, Y. Lahini, F. Pozzi, M. Sorel, R. Morandotti, and Y. Silberberg, Phys. Rev. Lett. 100, 170506 (2008).

[19] H. Schmitz, R. Matjeschk, Ch. Schneider, J. Glueckert, M. Enderlein, T. Huber, and T. Schaetz, Phys. Rev. Lett. 103, 090504 (2009).

[20] F. Zahringer, G. Kirchmair, R. Gerritsma, E. Solano, R. Blatt, and C. F. Roos, Phys. Rev. Lett. 104, 100503 (2010)

[21] B. C. Travaglione and G. J. Milburn, Phys. Rev. A 65, 032310 (2002).

[22] K. Karski, L. Foster, J.-M. Choi, A. Steffen, W. Alt, D. Meschede, and A. Widera, Science 325, 174 (2009).

[23] M. A. Broome, A. Fedrizzi, B. P. Lanyon, I. Kassal, A. Aspuru-Guzik, and A. G. White. Phys. Rev. Lett. 104, 153602 (2010).

[24] D. Lu, J. Zhu, P. Zou, X. Peng, Y. Yu, S. Zhang, Q. Chen, and J. Du, Phys. Rev. A 81, 022308 (2010).

[25] W. Dur, R. Raussendorf, V. M. Kendon, and H. J. Briegel, Phys. Rev. A 66, 052319 (2002).

[26] K. Eckert, J. Mompart, G. Birkl, and M. Lewenstein, Phys. Rev. A 72, 012327 (2005).

[27] C. M. Chandrashekar, Phys. Rev. A 74, 032307 (2006).

[28] Z.-Y. Ma, K. Burnett, M. B. d'Arcy, and S. A. Gardiner, Phys. Rev. A 73, 013401 (2006).

[29] V. Kendon and B. Tregenna, Phys. Rev. A 67, 042315 (2003).

[30] T. A. Brun, H.A. Carteret, and A. Ambainis, Phys. Rev. Lett. 91, 130602 (2003).

[31] V. Kendon, Math. Struct. in Comp. Sci. 17(6), 1169-1220 (2006).

[32] C. M. Chandrashekar, R. Srikanth and S. Banerjee, Phys. Rev. A. 76, 022316 (2007).

[33] S. Banerjee, R. Srikanth, C.M. Chandrashekar, and P. Rungta Phys. Rev. A, 78, 052316 (2008). 
[34] C. Liu and N. Petulante, Phys. Rev. E 81, 031113 (2010).

[35] R. Srikanth and S. Banerjee Phys. Rev. A 77, 012318 (2008).

[36] H. Ollivier and W. H. Zurek, Phys. Rev. Lett. 88, 017901 (2001).

[37] K. Modi, T. Paterek, W. Son, V. Vidal and M. Williamson, Phys. Rev. Lett. 10, 080501 (2010).

[38] S. Luo, Physical Review A 77, 022301 (2008).

[39] M. O. Scully and M. S. Zubairy, Quantum Optics (Cambridge University Press,1997).

[40] H.-P. Breuer and F. Petruccione, The Theory of Open Quantum Systems (Oxford University Press, 2002).

[41] C. M. Caves and B. L. Schumaker B L, Phys. Rev. A 31, (1985) 3068; B. L. Schumaker and C. M. Caves, Phys. Rev. A 31, (1985) 3093.

[42] J. D. Whitfield, C. A. Rodriguez-Rosario, and A. Aspuru-Guzik, Phys. Rev. A 81, 022323 (2010).

[43] M. Nielsen and I. Chuang, Quantum Computation and Quantum Information, Oxford (2000). 